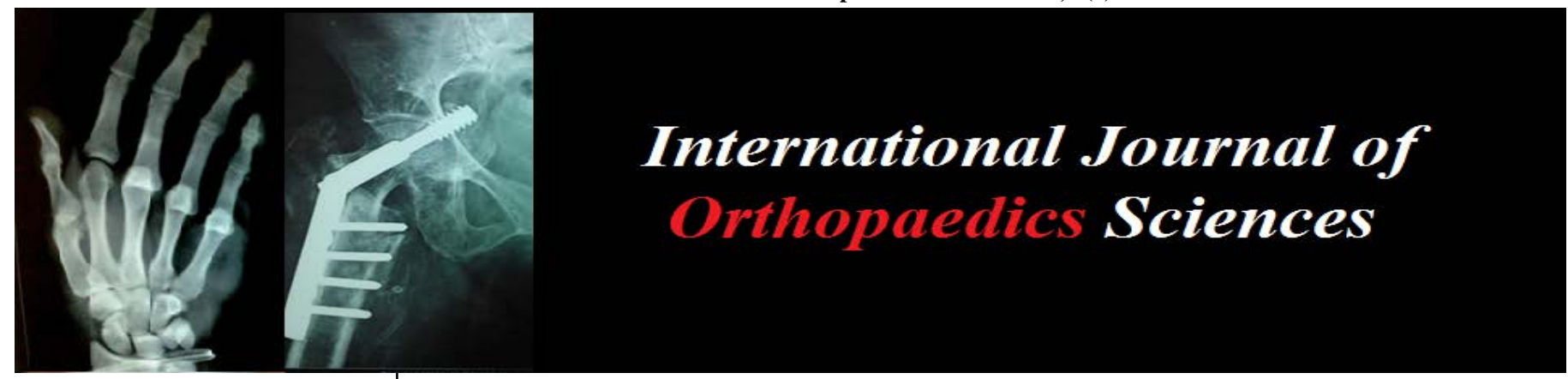

E-ISSN: 2395-1958

P-ISSN: 2706-6630

IJOS 2021; 7(4): 682-686

(C) 2021 IJOS

www.orthopaper.com

Received: 13-06-2021

Accepted: 19-07-2021

Ramesh Kumar Nagapuri Post Graduate, Department of Orthopaedics KAMSRC, LB Nagar, Hyderabad, Telangana, India

Dr. Prashanth Dhanraj

Associate Professor,

Orthopaedics, KAMSRC, LB

Nagar, Hyderabad, Telangana, India

Ehtesham Raza Sayeed Post Graduate, Department of Orthopaedics KAMSRC, LB

Nagar, Hyderabad, Telangana, India

Uday Kumar Goud Vallabhdas Post Graduate, Department of Orthopaedics KAMSRC, LB

Nagar, Hyderabad, Telangana, India

Ganta Venkata Sai Krishna Post Graduate, Department of Orthopaedics KAMSRC, LB Nagar, Hyderabad, Telangana, India

Syed Salar Ahmed

Post Graduate, Department of Orthopaedics KAMSRC, LB

Nagar, Hyderabad, Telangana, India

Corresponding Author: Dr. Prashanth Dhanraj Associate Professor, Orthopaedics, KAMSRC, LB Nagar, Hyderabad, Telangana, India

\section{Extra articular metadiaphyseal fractures of distal tibia managed by tip locking intramedullary Nailing: A case series}

\author{
Ramesh Kumar Nagapuri, Prashanth Dhanraj, Ehtesham Raza Sayeed, \\ Uday Kumar Goud Vallabhdas, Ganta Venkata Sai Krishna and Syed \\ Salar Ahmed
}

DOI: https://doi.org/10.22271/ortho.2021.v7.i4j.2951

\section{Abstract}

Introduction: Distal tibial fractures are commonest, often a result of high-energy trauma causing significant soft tissue injury and comminution. Being subcutaneous with tenuous soft tissue coverage it is poorly vascularized with more risk of infections, delayed union and non-union. This study is intended to know the efficacy of closed reduction with tip locking intramedullary nail, in distal extra articular metadiaphyseal tibia fractures with at least $4-5 \mathrm{~cm}$ of intact distal fragment.

Materials and Methods: This study is conducted on 18 skeletally mature patients, 14 male and 4 female, between December 2019 to December 2020 who fulfilled the inclusion criteria, using an Expert Tibial Nail System (ETNS)/tip locking IM nail. Patients were assessed clinically and radiologically at regular intervals for fracture union, functional ROM and any residual symptoms.

Results: Average time of fracture union was 15.2 weeks. The functional ROM in majority of patients was as pretrauma level at end of 15.2 weeks without any complications and residual symptoms on full weight bearing. Evaluated American Orthopaedic Foot and Ankle Society (AOFAS) ankle score was 80. Conclusion: ETNS/tip locking IM nail is an excellent option in tackling distal tibia fractures. It is a minimal invasive biomechanical procedure, giving stability even in osteoporotic bone. Minimal soft tissue complications with advantage of early fracture union and ambulation.

Keywords: Distal tibia fractures, intramedullary tip locking nail, expert tibia nail, fibula fixation

\section{Introduction}

Distal tibia fractures are commonest, often a result of high-energy trauma from axial and rotational force ${ }^{[3]}$ causing significant soft tissue injury, comminution and frequent association with swelling, echymossis, bruising, blebs (fracture blisters are a sign of massive soft tissue injury and a warning sign to delay surgical intervention, haemorrhagic blisters indicate underlying skin necrosis) and some extreme cases may be complicated by compartment syndrome and neurovascular damage. Being subcutaneous with limited muscle coverage on antero-medial aspect makes distal tibia poorly vascularized therefore more prone to infections, delayed union, non-union necessitating for secondary procedures and revision surgeries. Associated fibula fractures at same level or within $5-6 \mathrm{~cm}$ of ankle mortise jeoparadizes the ankle syndesmotic stability and needs stable fixation ${ }^{[4]}$. Several treatment modalities are available for management, both non-operative and operative each having its own advantages and complications. ILN amongst these has widened the range of indications in medullary osteosynthesis to include most of the tibial fracture patterns minimizing mean operation time, hospital stay, infections, surgical trauma to soft tissues, preserving fracture hematoma thus aiding in faster bone healing ${ }^{[6]}$, early mobilization and biomechanical stability. Expert Tibia Nailing System design has multiplanar locking options ${ }^{[8]}-5$ proximal and 4 distal with optimum Herzog's bend. The locking option at the tip of nail provides angular stability and has provision for compression at fracture site upto $7 \mathrm{~mm}$ by dynamic locking. It can be used as reamed/unreamed. All these features give it additional biomechanical stability than a conventional nail. 
The goal of treatment is to provide stable fixation by minimizing soft tissue damage, infections, deformity and avoiding joint stiffness with early union, restoration of full functional ROM to pretrauma levels without any residual symptoms.

\section{Material and methods}

It is a prospective study of extraarticular distal tibia fractures done in 18 skeletally mature patients, 14 male and 4 female, from December 2019 to December 2020 at our institute. The average age of patients is 37.8 years. Most common mode of injury was RTA. Ethical committee approval was taken prior to study. Informed consent taken from all patients included in the study. Radiograph of leg full length with ankle joint taken in AP and lateral view to evaluate fracture location, extent, comminution and any associated fibula fractures.

Associated fibula fracture within $7 \mathrm{~cm}$ of tibial plafond are fixed first with plating/rush nail, as this aids in obtaining length, alignment, stability of distal tibial segment. Stable fibular fixation reduces angular deviations and facilitates angular alignment and fracture stability which helps in better union rate.

\section{Inclusion Criteria}

Age $>18$ years, with unilateral distal tibia fractures.

Acute extraarticular distal tibia fractures with atleast $4-5 \mathrm{~cm}$ of intact distal fragment (to allow placement of atleast 2 distal locking screws at right angles) All closed and GustiloAnderson Grade-I fractures.

Segmental fractures with intact $4-5 \mathrm{~cm}$ of distal fragment.

Associated fibula fractures.

Fresh fractures within 1 week

\section{Exclusion Criteria}

Intraarticular fractures

Pathological fractures

Gustilo-Anderson Grade-II and Grade-III

Patients not suitable for spinal anaesthesia

Nerve injury

Non ambulating patients prior to injury

Patients not willing to give consent.

\section{Demographic Data}

\begin{tabular}{|c|c|}
\hline No of Patients & 18 \\
\hline Male & 14 \\
\hline Female & 4 \\
\hline Frequent Mechanism of injury - RTA & 13 \\
\hline Age & 37.8 Yrs \\
\hline Mean Operation Time & 83 min \\
\hline Mean Hospital stay & 4 days \\
\hline Mean Union Time & 15.2 weeks \\
\hline
\end{tabular}

After thorough examination and initial treatment patient is admitted. Fractured Limb is splinted with above knee POP slab. Limb elevation, LMWH, analgesics and for Grade-I fractures antibiotics are administered. Routine investigations performed, patient is evaluated for other comorbidities, anaesthesia fittness taken for Surgery. Skin condition is checked daily, if swelling, fracture blebs are present, then antiinflamatory drugs, limb elevation, ice pack application done and surgery is delayed till skin begins to wrinkle ${ }^{[2]}$. Open fractures are taken for surgery within 24 hours and closed fractures as soon as the skin condition allows.

Surgery is done under Spinal anaesthesia after giving antibiotic prophylaxis, in supine position with 90-110 degree knee flexion on a radiolucent fracture table and C-arm guidance. Preparation and draping is done in standard manner. Fracture reduced by manual traction, external manipulation and held in position after restoring length, axis and rotation.

Standard entry point taken, guide wire is passed till it crosses fracture line upto subchondral bone to lie at midpoint of talus [17], placing the nail at centre of wide metaphysis in AP and lateral views to avoid varus, valgus and AP tilt. Decision of reaming is taken per-operatively.

Appropriate size Expert Tibia Nail/tip locking nail is advanced till tip of subchondral bone.

Length, alignment, rotation are restored and locked more distally preferably at 3 sites if feasible or with atleast 2 screws one medio-laterally and another antero-posteriorly for better stabilization in close proximity to articular surface with free hand and proximally locked with an aiming device. Care taken not to leave empty locking holes at fracture site as there is high incidence of nail breakage at empty locking holes due to stress raising effect. Any associated fibula fractures within $5-6 \mathrm{~cm}$ of tibial plafond are fixed first with plate/rush nail as this aids in obtaining length, alignment of distal tibial segment. Mean operation time is 83 minutes. Average duration to surgery was 2 days, average hospital stay 4 days. Nail used was ETNS/ tip locking intramedullary nail. Postoperative biplanar radiographs taken for all cases. All patients received postoperative analgesics and antibiotics for 1week. Knee, ankle, toe ROM and quadriceps strengthening exercises started from 1st postoperative day. Dressing done on 2nd postoperative day wound assessed and patient is discharged. Patient was advised to visit on 14th day for woud assessment, suture removal and then at 6 weeks, 10 weeks, 14 weeks and 9 months. No weight bearing for 1st 6-8 weeks, then partial toe touch weight bearing allowed between 6-10 weeks and full weight bearing once the fracture unites clinically and radiologically. In each visit patient is assessed clinically for pain and tenderness at fracture site, surgical wound site infections, deformity, shortening, fracture stability, clinical union, functional ROM and radiologically for evidence of callus formation, atleast 3 cortices healing in both views is considered bone union.

Alignment measured in AP / Lateral radiographs from both legs. Shortening $>1 \mathrm{~cm}$ is significant, $>5^{\circ}$ valgus $/$ varus is malalignment, deformity is $>10^{\circ}$ procurvatum/recurvatum or $>10^{\circ}$ rotation ${ }^{[1]}$. American Orthopaedic Foot and Ankle Society (AOFAS) ankle score ${ }^{[19]}$ and ankle ROM are used to evaluate the outcome. Scoring system scales results in three major categories - Pain 40 points, Function 50 points and Alignment 10 points. Score between 90-100 are considered excellent, 75-89 is good, 50-74 fair and $<50$ poor. 


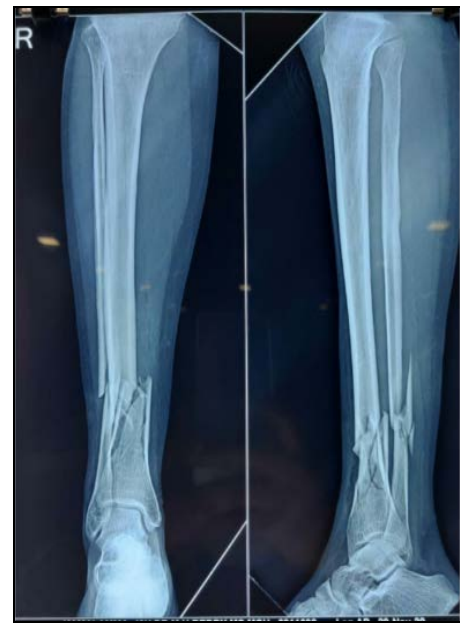

Pre-operative X-ray

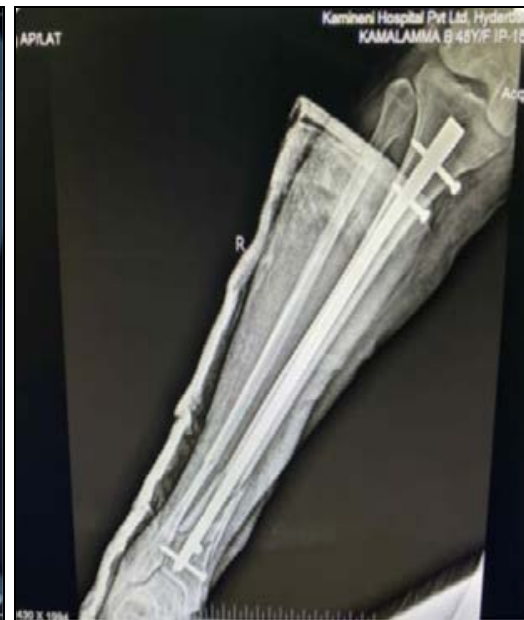

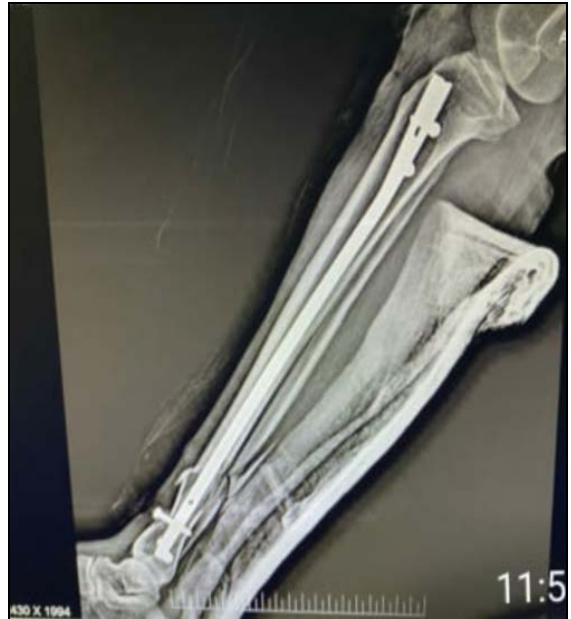

$11: 5$

\section{Results}

In this study all patients showed complete union clinically and radiologically with full functional ROM as pretrauma levels at about 15.2 weeks with no residual symptoms on full weight bearing in majority of patients. None of the patients developed any intraoperative or immediate postoperative complications related to procedure like embolism.

Fibula plating was done for 6 patients and rush nailing done in 1 patient in view of poor skin condition. 6 patients had knee pain at 14 weeks with occasional reccurence on prolonged walking, and difficulty walking on inclined surfaces/on using stairs, and 4 of them had mild decreased ankle ROM. None had any significant malalignment, 3 patients had less than $1 \mathrm{~cm}$ of shortening. Fracture site union was evident clinically and radiologically at end of 14 weeks in
Post-operative X-rays

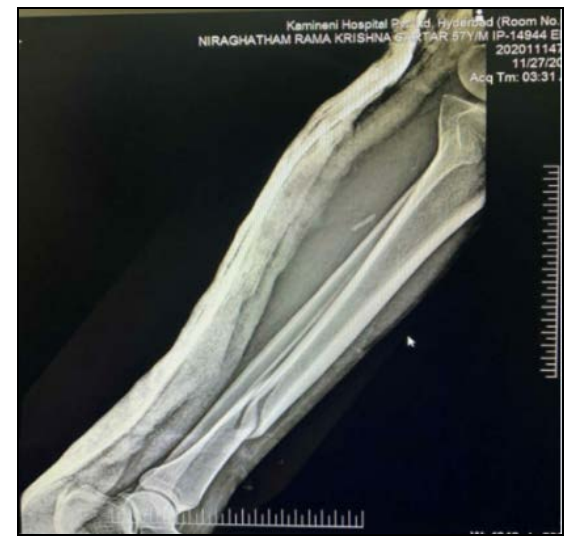

Pre-operative X-ray
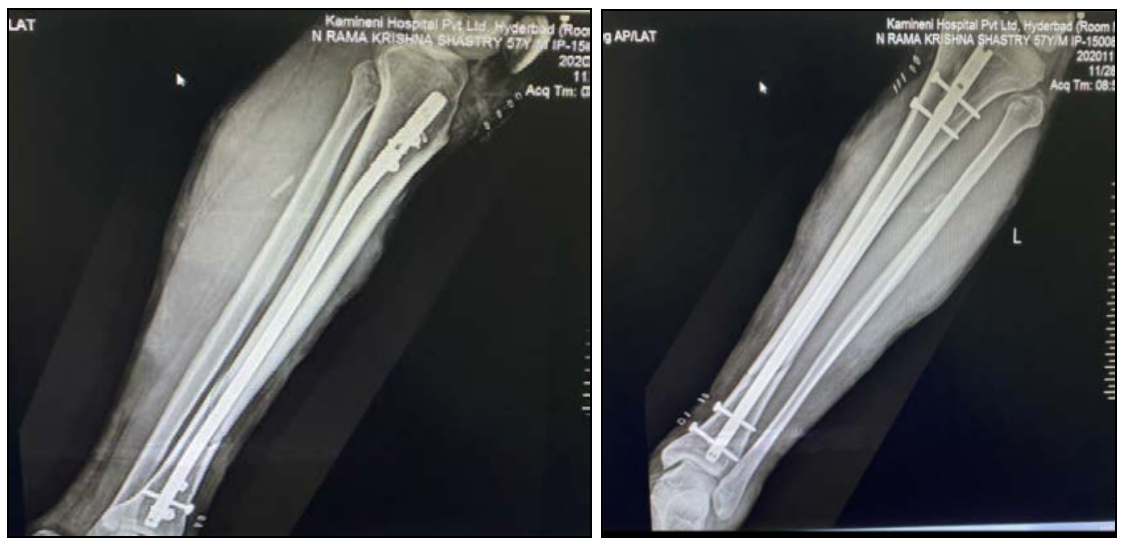

Immediate Post-operative X-rays

13 cases. 5 patients presented late for followup due to ongoing COVID pandemic, 4 patients at 18 weeks and 1 case at 20 weeks but at presentation all showed union clinically and radiologically. For those cases where fibula fixation was done also showed uneventfull union at same time as tibia. American Orthopaedic Foot and Ankle Society (AOFAS) ankle score and ankle ROM are used to evaluate the outcome. Average score was 80 points. Results were excellent in 12 patients, good in 2 patients, fair in 4 patients. The average pain score was 36 points, function score 34 points and alignment score 10 points with average ankle range of motion, 14 degrees dorsiflexion and 30 degrees of plantar flexion. Breakage of screws or proximal migration of nail was not seen in any of the cases. No incidence of infections (superficial/deep), delayed union or malunion.

\section{Discussion}

Treating distal tibia metadiaphyseal fractures possess several problems. Being subcutaneous antero-medially with tenous soft tissue coverage it is poorly vascularized in this region which increases the risk of infections, delayed union, nonunion [15]. Correcting translations, varus/valgus, procurvatum/recurvatum and rotational deformities are quite difficult. Several treatment modalities are available. Nonoperative treatment may be complicated by loss of reduction and subsequent malunion, delayed union, nonunion, joint stiffness due to prolonged immobilisation and cast complications, but can be used in stable fractures with severe comorbidities. In operative management selecting the implant (plating-supracutaneous or ORIF or MIPPO/external fixator/Ilizarov/IMILN) which best suits for given fracture pattern is debatable. MIPPO with locking plates reduces risk of extensive soft tissue dissection but is technically demading and anatomical reduction at fractured site is still difficult $[9,13]$ with risk of incomplete reduction, wound infections, secondary skin necrosis, skin irritation due to prominence of plate. External fixation may result in insufficient reduction, malunion, nonunion, ankle stiffness ${ }^{[20]}$ and pin tract infections. As opposed to plating which further compromises the vascularity of fractured fragments by surgical dissection and periosteal stripping we chose ILN as a viable option which preserves the fracture hematoma with minimal soft tissue injury giving biomechanically a stable construct which enhances early union with minimal complications ${ }^{[18]}$. Also plating can cause skin irritation due to subcutaneously lying hardware ${ }^{[16]}$, skin necrosis, soft tissue and wound site infections, wound dehisence, refracture ${ }^{[21]}$. As nail is a load sharing device early mobilization is possible, though restoring 
proper alignment of anatomical and mechanical axis without any deformity is of paramount significance in this procedure. Modern tibial nail designs enable more distal placement of screws in close proximity to the tip of nail, but the screws have less purchase in metaphyseal bone with increased stress on screws to maintain fracture alignment as compared to locking screws in diaphysis and diaphysiometaphyseal junction Intact fibula causes varus deformity and translation, making it difficult to hold and correct the distal fragment due to internal splintage. If fibula is fractured it needs to be fixed first. The overall functional outcome was excellent, score ranging about 80 points as per AOFAS with minimal residual symptoms in very few patients.

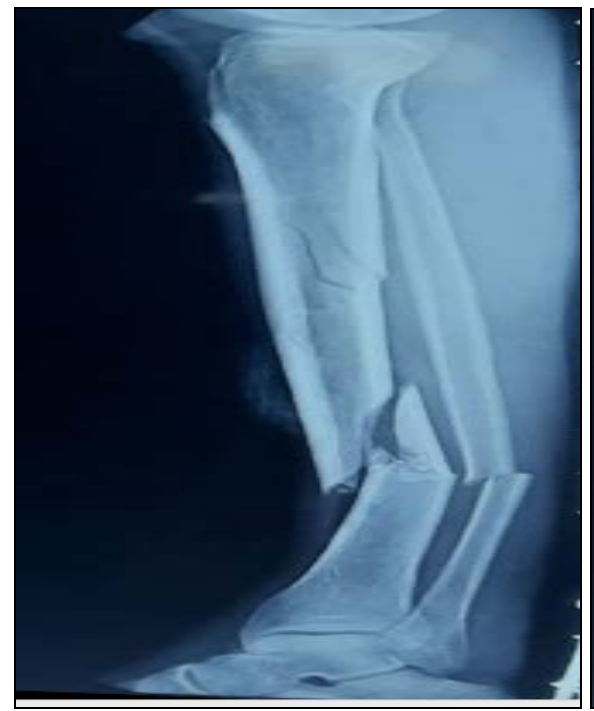

Segmental tibia with fibula fracture

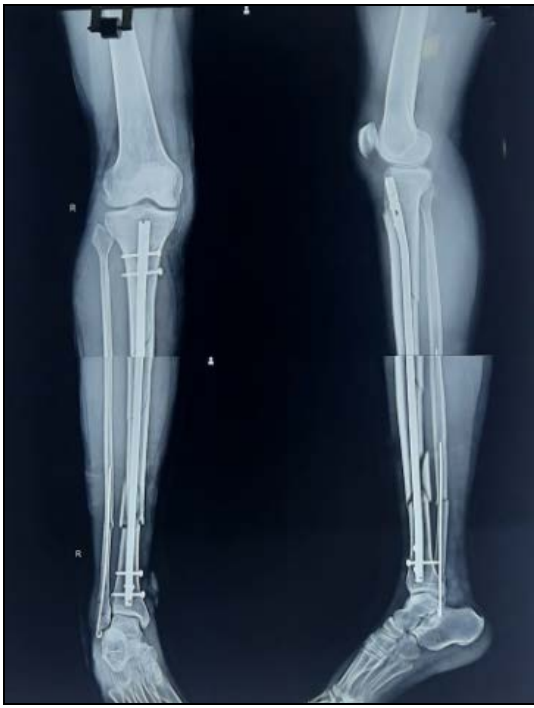

Immediate post-op X-ray

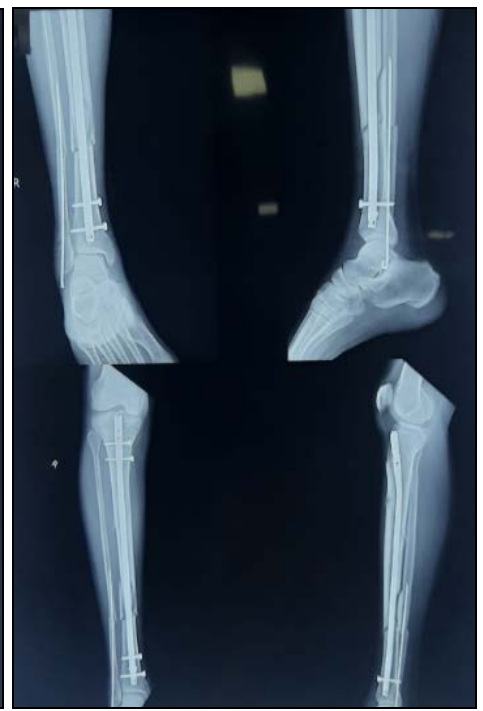

6 weeks post-operatively
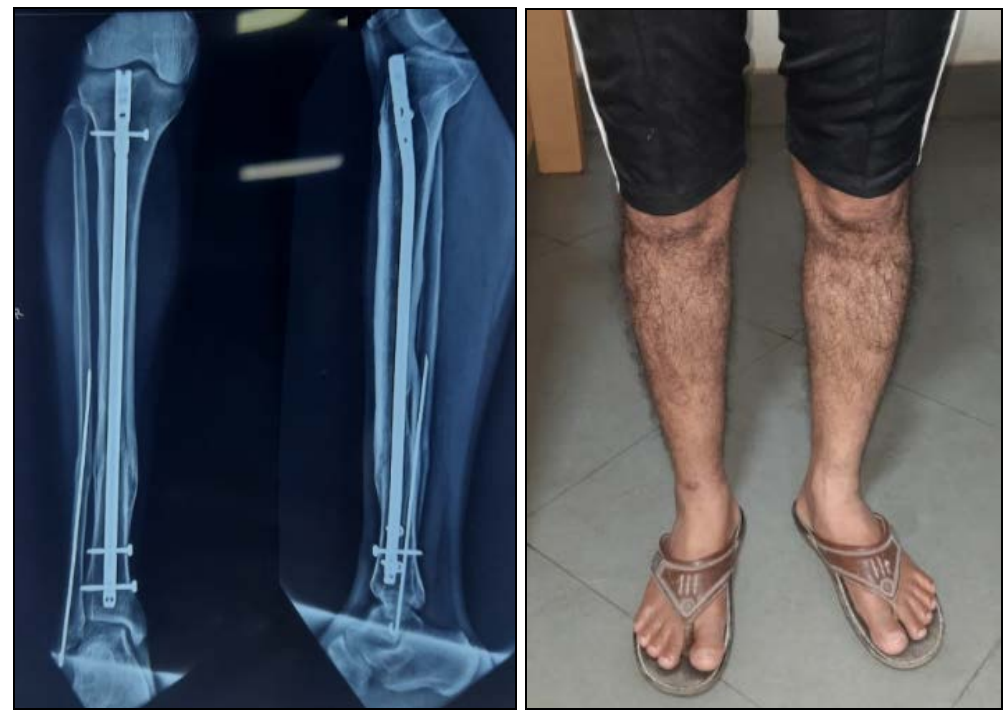

At 18 weeks Post-operatively showing union with restoration of length, alignment and rotation clinically and Radiologically.

\begin{tabular}{|c|c|}
\hline \multicolumn{2}{|c|}{ Osteosynthesis Data and Outcome analysis } \\
\hline Study & Prospective \\
\hline ETNS & 7 \\
\hline Distal Tip locking nail & 11 \\
\hline Fibula fixation & \\
\hline Plating & 6 \\
\hline Rush nail & 1 \\
\hline Mean Union Time & 15.2 weeks \\
\hline Nonunion & Nil \\
\hline AOFAS - Ankle score & 80 \\
\hline
\end{tabular}

\section{Conclusion}

IMIL nails with special design has distal tip locking option that can be effectively used in tackling the fractures in distal tibia. Being a minimally invasive and more biological technique with limited exposure and indirect reduction methods it avoids soft tissue and hardware complications. It also reduce the risk of delayed, nonunion, prevents knee and ankle stiffness, with early fracture union and patient mobilization as compared to other options of osteosynthesis in dealing with distal tibia fractures.

\section{References}

1. Mahajan NP et al. Role of tibia interlocking nail in treating distal tibial metadiaphyseal fractures Int J Res Orthop 2018;4(1):65-71.

2. Gawali SR, Kukale SB, Nirvane PV, Toshnival RO. Management of Fractures of Distal third Tibia by Interlocking Nailing. J Foot Ankle Surg (Asia-Pacific) 2016;3(1):15-22.

3. Richard RD, Kubaik E, Horwairtz DS. Techniques of surgical treatment of distal tibia fractures. Orthop clin North Am 2014;45:295-312.

4. Rene Attal et al. A multicenter case series of tibia fractures treated with Expert Tibia Nail (ETN) Arch 
Orthop Trauma Surg 2012;132:975-984.

5. Joveniaux P, Ohl X, Harisboure A, Berrichi A, Labatut L. Simon $\mathrm{P}$ et al. Distal tibia fractures: management and complications of 101 cases. Int Orthop 2010;34:583-8.

6. Hansen M, EI Attal R, Blum J, Blauth M, Rommens PM. Intramedullary Nailing of the Tibia with the Expert Tibia Nail. Oper Orthop Traumatol. 2009;21(6):620-35.

7. Vallier HA, Le TT, Bedi A. Radiographic and clinical comparisions of distal tibia shaft fractures (4 to $11 \mathrm{~cm}$ proximal to the plafond): plating versus intra-medullary nailing. J Ortho Trauma 2008;22(5):307-11.

8. Kuhn S, Hansen M, Rommens PM. Extemding the indications of intramedullary nailing with the Expert Tibial Nail. Acta Chir Orthop Traumatol Cech 2008;75(2):77-87.

9. Lau TW, Leung F, Chan CF, Chow SP. Wound complication of minimally invasive plate osteosynthesis in distal tibia fractures. Intl Orthop 2008;32:697-703.

10. Nork SE, Schwartz AK, Agel J, Schrick JL et al. Intramedullary nailing of distal metadiaphyseal tibial fractures. J Bone Joint Surg Am 2005;87(6):1213-1221.

11. Gorczyca JT, McKale J, Pugh K, et al. Modified tibial nails for treating distal tibia fractures. J Orthop Truma. 2002;16(1):18-22.

12. Francois J, Vandeputte G, Verheyden F, et al. Percutaneous plate fixation of fractures of distal tibia. Acta Orthop Belg 2004;70(2):148-154.

13. Borg T, Sune L, Ulf L. Percutaneous plating of distal tibia fractures; preliminary results in 21 patients. Injury 2004;35:608-14.

14. Kumar A, Charlebois SJ, Cain EL, Smith RA, Daniels AU, Crales JM. Effect of fibular plate fixation on rotational stability of simulated distal tibial fractures treated with intramedullary nailing. J Bone Joint Surg Am 2003;85-A(4):604-608.

15. Blick SS, Brumback RJ, Lakatos R, et al. Early prophylactic bone grafting of high energy tibial fractures. Clin Orthop Relat Res 1989;(240):21-41.

16. Puno RM, Teynor JT, Nagano J, Gustilo RB. Critical analysis of results of treatment of 201 tibial shaft fractures. Clin Orthop 1986;212:113-21.

17. Tyllianakis $M$, Megas $P$, Giannikas D. Interlocking intramedullary nailing in distal tibial fractures Orthop 2000;23(8):805-808.

18. Court-Brown CM, Will E, Christie J. Mc Queen MM. Reamed or unreamed nailing for closed tibial fractures. A prospective study in Tscherne CI fractures. J Bone Joint Surg Br 1996;78:580-583.

19. Kitaoka HB, Alexander IJ, Adelaar RS, Nunley JA, Meyerson MS, Sanders M. Clinical rating systems for the ankle Hind foot, mid foot, hallux and lesser toes. Foot Ankle Int 1994;15:349-353.

20. Thomas A, Russel J, Charles T, David G, Lavelle. Fractures of Tibia and fibula Rockwood and Green's Fractures in Adults, $3^{\text {rd }}$ Edition. J. B. Lipincott Company, Philadelphia. 1991; 2:1915-82.

21. Wiss DA. Flexible medullary nailing of acute tibial shaft fractures. Clin Orthop 1986;212:122-32. 